Revue d'histoire de l'Amérique française

Q8. REVUE D'HISTOIRE DE L'AMÉRIQUE FRANÇAISE

\title{
Opinions en histoire
}

\section{Roland Lamontagne, Pierre Chaunu et Frédéric Mauro}

Volume 21, numéro 2, septembre 1967

URI : https://id.erudit.org/iderudit/302666ar

DOI : https://doi.org/10.7202/302666ar

Aller au sommaire du numéro

Éditeur(s)

Institut d'histoire de l'Amérique française

ISSN

0035-2357 (imprimé)

1492-1383 (numérique)

Découvrir la revue

Citer ce document

Lamontagne, R., Chaunu, P. \& Mauro, F. (1967). Opinions en histoire. Revue

d'histoire de l'Amérique française, 21(2), 181-184.

https://doi.org/10.7202/302666ar d'utilisation que vous pouvez consulter en ligne.

https://apropos.erudit.org/fr/usagers/politique-dutilisation/ 


\section{OPINIONS EN HISTOIRE}

\section{PROBLEMES DE MÉTHODE: le structuralisme en histoire}

Dans les domaines des sciences sociales, des études littéraires et de la philosophie, les problèmes de structuralisme suscitent de nouvelles recherches; en raison de leur complexité, ils se situent à un carrefour interdisciplinaire. Les études historiques sous-tendent les sciences de l'homme. Sous les multiples aspects de l'espace-temps, de la dynamique d'ensemble, de concepts et de schémas nouveaux, il est bien naturel qu'elles soient l'objet de débats d'ordre structural ou encore structurel, selon certains textes.

Mise en œuvre à Montréal, cette méthode structuraliste d'approche historique s'inscrit dans un domaine de recherche et de collaboration internationale, notamment, de l'École des Annales E.S.C., de Fernand Braudel, du Centre International de Synthèse et de personnalités éminentes du savoir historique en France et au Brésil, entre autres, Pierre Chaunu, Frédéric Mauro, René Taton, Gilberto de Mello Freyre. Ces enquêtes historiques acheminent à une formulation d'éléments de méthode que renferme cette note brève. L'histoire dite structurale constitue à la fois une vision du monde et une méthode de travail, liées à l'interdépendance de toutes les composantes de la vie collective, dans une dynamique d'ensemble de l'espace-temps, à l'exemple de la Méditerranée de Braudel, des "Océans" de Chaunu et de Mauro. Au carrefour de méthodes interdisciplinaires, elle analyse les variables influençantes, influencées et les résultantes, susceptibles d'un nombre indéterminé d'interprétations, dans une recherche de causalité indéfiniment hors d'atteinte. Le structuralisme en histoire enrichit nos méthodes d'approche de la condition humaine. Dans un excellent esprit de collaboration, Pierre Chaunu et Frédéric Mauro apportent ici d'utiles précisions relatives à l'histoire sérielle et au thème: histoire et structure.

Université de Montréal

ROLAND LAMONTAGNE

\section{[181]}




\section{L'HISTOIRE SERIELLE}

L'histoire a muté au cours des trente dernières années.

Pour bien comprendre ce que nous entendons par histoire sérielle, il faut partir, évidemment, de l'histoire positiviste du début du XXe siècle. Un point de perfection est atteint considéré comme point d'arrivée. L'histoire positiviste, un merveilleux outil qu'il faut bien se garder de laisser rouiller. Elle pousse à son point d'authentique perfection les méthodes critiques d'établissement du texte et du fait, au terme d'une trajectoire qui part de l'humanisme critique de Laurent Valla, se développe majestueusement avec les bénédictins de Saint Maur, arrive proche du sommet, avec la critique historique allemande de la seconde moitié du XIXe siècle. Un merveilleux outil; pour quoi faire?

Pour rien. Pour collectionner des faits, arbitrairement et inconsciemment privilégiés, pour perfectionner, si l'on veut, la vieille chronique: une chronique élargie, du Prince jusqu'à l'Etat.

Le tournant de l'histoire, c'est accessoirement, le tournant de l'histoire totale, mais c'est, bien plus essentiellement, le tournant de l'histoire science auxiliaire privilégiée des sciences humaines. L'histoire sérielle, la dimension dans le temps de toutes les sciences de l'homme. Il n'y a pas de science sans mesure. C'est pourquoi le progrès des sciences humaines est aussi conditionné par la mise en place d'un important appareil statistique. Du même coup, la plupart des sciences humaines sont enfermées dans le temps court de l'ère statistique, mieux même dans le temps très court des dernières décennies, celles, si l'on veut, d'une statistique perfectionnée et ambitieuse de totalité.

L'histoire sérielle, dans cette perspective, est d'abord, l'humble artisane qui prolonge à travers l'épaisseur pluriséculaire de l'ère préstatistique, antéstatistique et protostatistique, les séries sur lesquelles travaillent les sciences de l'homme du présent, de la démographie à l'économique, d'une sociologie des rapports grossiers de production et de consommation, au monde plus subtile et tout aussi essentiel des représentations. Après le traitement massif des mercuriales, des archives fiscales des ports, après l'utilisation des registres paroissiaux, des cadastres,... des alcabalas... et des compoix, voici les possibilités illimitées de la sémantique quantitative appliquée à l'imprimé, mais, plus récemment, aux masses dormantes des archives religieuses et para-religieuses. Chaque série documentaire retenue, chaque élément destiné à nourrir la série, est, naturellement, soumis à l'instrument critique traditionnel. Mais une fois constituée la série porte en elle ses propres raisons. C'est pourquoi, nous l'avons dit, le document parle deux fois, une première fois comme témoin isolé, une seconde fois beaucoup plus clairement, à l'intérieur de la série constituée.

Voilà pourquoi nous disons plus volontiers histoire sérielle. Histoire sérielle plus et mieux qu'histoire quantitative. Les deux notions bien sûr, se recoupent partiellement. La notion d'histoire quantitative est plus restreinte mais elle est plus ambitieuse. Réservons-la aux périodes et aux approches, évidemment, économiques qui permettent une quantification totale, la constitution d'un système du type comptabilité nationale ou régionale. La série implique toute quantification même partielle: c'est dire qu'elle déborde largement le champ de l'économique. Une quantification moins poussée mais qui entreprend des remontées de plus en plus nombreuses, de plus en plus hardies, dans des secteurs jusqu'alors totalement abandonnés de la connaissance du passé. 
C'est dire les dimensions du propos. Modeste auxiliaire, au départ, l'approche sérielle en histoire déborde rapidement le champ étroit de la technique. L'histoire sérielle implique structuration historique des sciences de l'homme. Une approche scientifique, de l'homme en société, coupée de la dimension temporelle, coupée des déroulements séculaires que l'histoire, seule, peut atteindre n'est qu'une vaine tentative nécessairement vouée à l'échec. Science auxiliaire polytechnicienne de toutes les sciences humaines, à leur tour servantes de l'histoire, l'histoire sérielle accomplit volontiers une mission fédératrice dans la grande famille des sciences humaines. Cette approche est typiquement française. Elle doit beaucoup à une conjoncture particulière des années 30 et 40 qu'il serait trop long d'expliquer. Une attention toute particulière à l'histoire, à la démographie et à la géographie, au temps donc, au nombre des hommes et à leur dynamique, à une dialectique raisonnée de l'homme et de l'espace-temps, l'espace-temps résultante du paysage...., telle est la caractéristique la plus profonde, aujourd'hui, peut-être, de l'école française ès sciences humaines.

Là notre richesse, là aussi la racine d'un difficile dialogue avec les puissantes écoles nord américaines attentives à d'autres aspects, maîtresses d'autres techniques.

C'est dire la place de l'histoire sérielle, aujourd'hui et demain, dans le champ en expansion des sciences sociales.

Université de Caen

Pierre Chaunu

\section{HISTOIRE ET STRUCTURE}

La méthode structurelle si en vogue chez les historiens depuis la fin de la Seconde guerre Mondiale et le triomphe de l'école des Annales, de Fernand Braudel, ne prétend pas être autre chose qu'une problématique utile, un instrument de travail. Elle repose sur l'idée que les civilisations, dont la succession forme l'histoire même de l'humanité, représentant des ensembles à la fois politiques, économiques, sociaux, culturels qui ont leurs lois propres, leurs "structures" propres qu'il s'agit de découvrir - que la succession de ces civilisations et le passage continu ou discontinu de l'une à l'autre se font à travers les rythmes divers - courts ou longs - de la conjoncture - qu'il faut préciser ces rythmes et les expliquer - que les structures qu'ils engendrent peuvent être reconstitués sous formes de modèles comparables entre eux et comparables aux modèles du présent qu'ils expliquent et permettent de mieux définir et que l'histoire, ainsi, est utile, au sens le plus noble du mot.

D'une conception événementielle attachée surtout à l'éphémère, à ce que "jamais on ne verra deux fois", les historiens sont passés à cette conception conjoncturelle et structurelle de leur discipline. Faisant le chemin en sens inverse les économistes et les sociologues sont allés d'une théorie abstraite et universelle à une théorie relative, où les lois ne sont plus générales mais propres à une structure donnée et que l'on a appelée, d'un mot nord-américain, l'institutionalisme. On aurait pu dire le structuralisme, le mot institution en français désignant plutôt la surface des choses, et le mot structure les réalités en profondeur (Nous distinguons, dans le sens de cette gradation: droit, institutions, structures). L'histoire et l'économie se sont rencontrées à mi-chemin dans l'étude de la structure économique - et l'histoire et la sociologie dans celle de la structure 
sociale. Ainsi les différentes sciences sociales vont vers une unité chaque jour plus grande et l'histoire est une de ces sciences; elle est même l'ensemble des autres appliqué au passé: elle ne diffère des autres que parce qu'elle est rétrospective alors que les autres sont prospectives.

L'anthropologie culturelle a rencontré elle aussi la structure et l'élément anthropologique est essentiel à la compréhension d'une civilisation déterminée. Il semble que le terme même de structure ait été employé par les ethnologues avec la signification de très longue durée qu'elle n'a pas forcément en histoire ou en économie. Elle rejoint presque l'idée d'une nature humaine chère aux classiques.

On peut donc se poser deux questions:

$1^{\circ}$ ) Au-delà des structures politiques, économiques, sociales et mentales, l'historien ne doit-il pas chercher à faire la part de ce qui est plus permanent et qui relève en fin de compte de la condition humaine ? En d'autres termes ne doit-il pas pour mieux distinguer les structures, faire la part de ce qui est la nature ?

$\left.2^{\circ}\right)$ Est-il permis aux spécialistes des différentes sciences sociales, anthropologues compris, de s'engager sur la voie de la philosophie et même de la métaphysique et d'accorder à ces structures une essence et une existence dépassant les simples limites du concept construit et de l'hypothèse de travail ? Faut-il, comme Foucault, croire à des structures distinctes de l'homme et auxquelles l'homme se contente de participer?

En ce qui nous concerne, nous pensons qu'il faut laisser aux philosophes le soin d'écrire une nouvelle métaphysique. Mais qu'ils attendent un peu encore les progrès des sciences sociales. Leur temps n'est pas encore venu.

Université de Toulouse

FrÉdÉric MaUro 